\title{
FITOSSOCIOLOGIA EM COMUNIDADES FLORESTAIS DO PROJETO RADAMBRASIL NO BIOMA AMAZÔNIA
}

\author{
PHYTOSOCIOLOGY IN FOREST COMMUNITIES OF RADAMBRASIL PROJECT IN THE \\ AMAZON BIOME
}

\author{
Luani Rosa de Oliveira Piva ${ }^{1}$, Carlos Roberto Sanquetta ${ }^{2}$, Jaime Wojciechowski ${ }^{3}$, \\ Ana Paula Dalla Corte 4 \\ 1, 2, 3,4 Universidade Federal do Paraná, Curitiba, Paraná, Brasil - luanipiva@yahoo.com.br, \\ carlossanquetta@gmail.com,jaimewo@gmail.com \&anapaulacorte@gmail.com
}

\begin{abstract}
RESUMO
A Floresta Amazônica é o maior hotspot de biodiversidade de seres vivos do planeta, incluindo espécies de plantas. Portanto, informações sobre a estrutura da composição florística dessa floresta - obtidas por meio de análises fitossociológicas - são de sua importância. A hipótese de estudo é que a diversidade florística na Amazônia, observada a partir de levantamentos florestais realizado nas décadas de 70 e 80 , assemelha-se à diversidade encontrada em levantamentos datados dos últimos 10 anos. 0 objetivo da presente pesquisa foi avaliar parâmetros fitossociológicos da estrutura horizontal em comunidades florestais do Projeto RADAMBRASIL. Foram analisados dados para duas tipologias florestais predominantes no Bioma Amazônia: Floresta Ombrófila Densa (1.158 parcelas; 70.371 árvores) e Floresta Ombrófila Aberta (854 parcelas; 43.860 árvores). Os dados foram extraídos da plataforma online BDiA (Banco de Dados de Informações Ambientais), criada pelo IBGE (Instituto Brasileiro de Geografia e Estatística). Sete parâmetros da estrutura horizontal foram calculados e avaliados, além do Índice de Shannon-Wiener $\left(\mathrm{H}^{\prime}\right)$. Os resultados indicaram a ocorrência de 76 famílias e 661 espécies de árvores, em Floresta Densa. Já para Floresta aberta, foram 69 famílias e 593 espécies. Uma alta diversidade florística pôde ser observada nas parcelas de Floresta Densa e Floresta Aberta, expressa pelos valores de $\mathrm{H}^{\prime}$ encontrados, iguais a 5,33 e 5,36 nats.ind ${ }^{-1}$, respectivamente. As famílias com maior número de indivíduos, em ambas as tipologias, foram Lecythidaceae, Sapotaceae e Burseraceae. As espécies Eschweilera coriacea (DC.) S.A.Mori, Pouteria caimito (Ruiz \& Pav.) Radlk. e Protium altissimum (Aubl.) Marchand, apresentaram os maiores valores de importância (VI) nas tipologias analisadas. Além disso, essas espécies destacaram-se pelos altos valores de dominância absoluta e frequência absoluta. Concluiu-se que a diversidade florística na Floresta Amazônica, observada a partir de levantamento realizado nas décadas de 70 e 80, assemelha-se à diversidade encontrada em levantamentos datados dos últimos 10 anos.
\end{abstract}

PALAVRAS-CHAVE: Análise fitossociológica, Composição florística, Estrutura florística, Floresta Amazônica.

\section{ABSTRACT}

The Amazon rainforest is the most biologically diverse place of living beings on Earth, including plant species. Therefore, information about floristic composition and vegetation structure of this rainforest - obtained through phytosociological analysis - is extremely important. The study hypothesis is that the floristic diversity in the Amazon, observed from forest surveys carried out in the $70 \mathrm{~s}$ and $80 \mathrm{~s}$, is similar to the diversity found in surveys dating from the last 10 years. This study aimed to analyze phytosociological parameters of the horizontal structure in forest communities of RADAMBRASIL Project. Data were analyzed for two forest types in the Amazon Biome: Ombrophilous Dense Forest (1,158 plots; 70,371 trees) and Ombrophilous Open Forest ( 854 plots; 43,860 trees). Data were obtained from the Environmental Information Database (BDiA), a web platform created by the Brazilian Institute of Geography and Statistics (IBGE). Seven parameters of the horizontal structure of the vegetation were calculated, in addition to the Shannon-Wiener Index $\left(\mathrm{H}^{\prime}\right)$. The results indicated the occurrence of 76 families and 661 species of trees, for Ombrophilous Dense Forest data. For Ombrophilous Open Forest, 69 families and 593 species. A high floristic diversity was observed for both forest types, Dense Forest and Open Forest; the $\mathrm{H}^{\prime}$ values were 5.33 and 5.36 nats.ind $^{-1}$, respectively. The families with the largest number of individuals, in both forest types, were Lecythidaceae, Sapotaceae and Burseraceae. The species Eschweilera coriacea (DC.) S.A.Mori, Pouteria caimito (Ruiz \& Pav.) Radlk. and Protium altissimum (Aubl.) Marchand presented the highest importance value, for both forest types analyzed. Also, these species stood out for their high values of absolute dominance and absolute frequency. As conclusion, the floristic diversity in the Amazon forest, observed from surveys carried out in the $70 \mathrm{~s}$ and $80 \mathrm{~s}$, is similar to the diversity found in recent surveys.

KEYWORDS: Phytosociological analysis, Floristic composition; Floristic structure, Amazon rainforest. 


\section{INTRODUÇÃO}

A Floresta Amazônica é a maior floresta tropical úmida da Terra, sendo considerada a região de maior biodiversidade de espécies animais e vegetais (TER STEEGE et al., 2016). Com relação a espécies de plantas arbóreas, estima-se a ocorrência de, aproximadamente, 16.000 ao longo da bacia Amazônica (TER STEEGE et al., 2013). Entretanto, é de conhecimento que a Floresta Amazônica brasileira vem sofrendo, ao longo das últimas décadas, processos intensivos de conversão da floresta em outros usos, como agricultura e pastagem (MACEDO et al., 2012; NEPSTAD et al., 2014), os quais impactam negativamente na composição da biodiversidade desse ecossistema.

Dessa forma, levantamentos florísticos para avaliar a biodiversidade de espécies de árvores na Amazônia são de suma importância. Maiores conhecimentos acerca da diversidade florística da Amazônia darão suporte a estratégias mais eficientes de conservação e restauração desse bioma (ORME et al., 2005).

O Projeto RADAMBRASIL foi pioneiro no levantamento de informações dos recursos naturais brasileiros, incluindo o bioma Amazônia, por meio de levantamentos aéreos conduzidos nas décadas de 70 e 80 . Recentemente, o Instituto Brasileiro de Geografia e Estatística (IBGE) lançou a plataforma "Banco de Dados de Informações Ambientais" (BDiA), na qual é possível ter acesso aos dados do RADAMBRASIL, incluindo informações dos inventários florestais conduzidos no âmbito do Projeto (IBGE, 2019).

Na plataforma BDiA, dados de 25 volumes publicados pelo Projeto RADAMBRASIL estão disponibilizados online, de tal maneira que o usuário pode acessar e compartilhar, de forma interativa, todo o acervo de informações ambientais do IBGE. Com isso, foi possível a obtenção dos dados utilizados no presente artigo.

Diversos estudos relevantes, que elucidaram a biodiversidade e composição florística na Floresta Amazônica, foram conduzidos ao longo dos últimos 30 anos. Alguns podem ser destacados, como é o caso de Hopkins (2007, 2019), Milliken et al. (2011), e Ter Steege et al. (2013).

Ter Steege et al. (2013), além de explanarem a quantidade de espécies de árvores na Floresta Amazônica (cerca de 16.000), introduziram o conceito de "hiperdominância" de famílias e espécies de árvores. Segundo esses autores, espécies como Eschweilera coriacea (DC.) S.A.Mori, Licania apetala (E.Mey.) Fritsch e Euterpe oleracea Mart., além das famílias Arecaceae, Myristicaceae e Lecythidaceae, são consideradas "hiperdominantes" na bacia Amazônica, ou seja, correspondem à metade de todas as árvores existentes na Amazônia. Além disso, os autores descreveram que cerca de 5.000 espécies são consideradas muito raras, e alertaram que estas correm risco de extinção antes mesmo de serem observadas e descritas.

Hopkins (2007, 2019) questionou que há subestimativas no número de espécies de plantas e biodiversidade na Amazônia. Para corrigir esses valores, o autor afirmou que maiores investimentos nas coletas de plantas, com exemplares de qualidade, são imprescindíveis. Ainda, essas subestimativas podem limitar as estratégias de conservação, especialmente de espécies consideradas raras (HOPKINS, 2007, 2019).

Milliken et al. (2011) mencionaram que a falta de informações sobre a distribuição das espécies ao longo dos gradientes ambientais na Amazônia, bem como desconhecimentos da diversidade filogenética, ainda são obstáculos para a elaboração de estratégias de conservação da biodiversidade dessa floresta.

Análises dos parâmetros fitossociológicos vêm sido conduzidas na Amazônia, de tal forma a se obter mais informações sobre os padrões de distribuição de espécies de plantas, a estrutura das comunidades florestais, bem como da diversidade florística. Por exemplo, Oliveira et al. (2008) e Silva et al. (2015) realizaram estudos florísticosestruturais na Amazônia Central, enquanto os estudos de Santana et al. (2004) e Pinheiro et al. (2007) são exemplos na Amazônia Oriental.

A hipótese de estudo que a diversidade florística na Amazônia, observada a partir de levantamentos florestais realizado nas décadas de 70 e 80 , assemelha-se à diversidade encontrada em levantamentos datados dos últimos 10 anos.

Diante do exposto, o objetivo do presente trabalho foi avaliar parâmetros fitossociológicos da estrutura horizontal em comunidades florestais do Projeto RADAMBRASIL, análise inédita para dados de parcelas localizadas nos estados que compõem o bioma Amazônia.

\section{MATERIAL E MÉTODOS}

\section{Base de dados}

Os dados foram extraídos da plataforma digital BDiA de acesso livre e disponível em https://bdiaweb.ibge.gov.br/. Na área temática "Vegetação" do BDiA, é possível extrair dados brutos, por estado, de inventários florestais conduzidos no âmbito do Projeto RADAMBRASIL, nas décadas de 70 e 80 . Portanto, essa plataforma contempla a maior base de dados até hoje 
coletada para o bioma Amazônia.

A base de dados utilizada no presente artigo é oriunda dos inventários florestais desenvolvidos ao longo do Projeto RADAMBRASIL, para indivíduos arbóreos de grande porte, ou seja, com diâmetro à altura do peito (DAP) $\geq 30$ $\mathrm{cm}$. Nesses dados, constam com informações das identificações botânicas, em nível de família e espécie, além do local do inventário (estado) e número de identificação de cada parcela.

Foram selecionados dados para as duas tipologias florestais predominantes no bioma Amazônia: Floresta Ombrófila Aberta e Floresta Ombrófila Densa (Tabela 1). A base de dados resultante é composta por 114.231 árvores, distribuídas em 2.012 parcelas (um hectare cada), nos 9 estados brasileiros que compõem o bioma Amazônia: Acre, Amapá, Amazonas, Maranhão, Mato Groso, Pará, Rondônia, Roraima e Tocantins.

Tabela 1. Número de indivíduos e número de parcelas em áreas do bioma Amazônia, de acordo com a tipologia florestal.

\begin{tabular}{ccc}
\hline Tipologia & No árvores & № parcelas \\
\hline Floresta Ombrófila Densa & 70.371 & 1.158 \\
Floresta Ombrófila Aberta & 43.860 & 854 \\
\hline Total & $\mathbf{1 1 4 . 2 3 1}$ & $\mathbf{2 . 0 1 2}$ \\
\hline
\end{tabular}

\section{Análise fitossociológica}

Os seguintes parâmetros fitossociológicos da estrutura horizontal foram calculados, para cada uma das tipologias florestais estudadas (LAMPRECHT, 1964; MUELLER \& ELLENBERG, 1974; MARTINS, 1991):

1. Densidade absoluta (DA): diz respeito ao número de indivíduos total de uma mesma espécie por unidade de área. Para obter a DA é necessário o conhecimento do número de indivíduos amostrados da espécie $\left(n_{i}\right)$ e da área total amostral $(A)$ :

$$
D A=\frac{n_{i}}{A}
$$

2. Densidade Relativa (DR): revela, em porcentagem, a participação de cada espécie em relação ao número total de indivíduos de todas as espécies. É calculada pela razão de DA de cada espécie pela densidade total (soma dos valores de DA para cada espécie):

$$
D R=\frac{D A}{\sum_{i=1}^{j} D A} * 100
$$

Em que: $\mathrm{j}$ = número total de espécies observadas.
3. Dominância absoluta (DoA): representa o espaço (área) ocupada por determinada espécie, expressa por unidade de área $\left(\mathrm{m}^{2} \cdot \mathrm{ha}^{-1}\right)$. Para obter a dominância absoluta é necessário o cálculo prévio das áreas transversais $\left(\mathrm{g}_{\mathrm{j}}\right)$ das árvores. Em seguida, a área basal da espécie $\left(G_{i}\right)$ é obtida pela soma das gj para cada espécie. Por fim, a DoA da espécie é calculada pela razão da Gi pela área total amostrada (A):

$$
\left\{\begin{array}{l}
g_{j}=\frac{\pi}{40.000} * D A P_{j}^{i} \\
D o A=\frac{\sum_{j=1}^{i} g_{j}}{A}=\frac{G_{i}}{A}
\end{array}\right.
$$

4. Dominância relativa (DoR): é calculada pela razão da dominância absoluta de cada espécie (DoA) pela dominância total (soma dos valores de DoA para cada espécie):

$$
D o R=\frac{D o A}{\sum_{i=1}^{j} D o A} * 100
$$

5. Frequência absoluta (FA): indica a porcentagem (ou proporção) de ocorrência de uma espécie em uma determinada área. A frequência absoluta de cada espécie é calculada pela razão do número de unidades amostrais $\left(U_{i}\right)$ onde foram encontradas a espécie e o número total de unidades amostrais $\left(U_{T}\right)$ :

$$
F A=\frac{U_{i}}{U_{t}} * 100
$$

6. Frequência relativa (FR): é calculada pela razão da frequência absoluta de cada espécie (FA) pela frequência total (soma dos valores de FA para cada espécie):

$$
F R=\frac{F A}{\sum_{i=1}^{j} F A} * 100
$$

7. Valor de importância (VI): resulta da soma da densidade, dominância e frequência relativa ( $D R$, DoR e FR, respectivamente) de cada espécie:

$$
V I=D R+D o R+F R
$$

Ademais, a diversidade florística também foi avaliada por meio do Índice de Shannon-Wiener $\left(H^{\prime}\right)$ (SHANNON \& 
WIENER, 1948):

$$
H^{\prime}=-\sum_{i=1}^{j} p_{i} * \ln p_{i}, \quad p_{i}=\frac{n_{i}}{N}
$$

Em que: $j$ = número total de espécies observadas; $p_{i}=$ proporção de indivíduos da i-ésima espécie; In = logaritmo de base neperiano; $\mathrm{n}_{\mathrm{i}}=$ número de indivíduos amostrados para a espécie i; $\mathrm{N}=$ número total de indivíduos amostrados.

Todos os parâmetros foram calculados a partir da linguagem $R$ ( $R$ CORE TEAM, 2019). Para tanto, foi utilizada a função "fitoR" (DALAGNOL et al., 2016), para cálculo dos descritores fitossociológicos, e o pacote "ggplot2" (WICKHAM, 2016), para elaboração das figuras.

\section{RESULTADOS E DISCUSSÃO}

\section{Floresta Ombrófila Densa}

Para os dados de Floresta Ombrófila Densa, observa-se que a distribuição de indivíduos, de acordo com o DAP, concentra-se nas classes de diâmetro entre $30-70 \mathrm{~cm}$ (Figura 1). Ao total, 66.784 indivíduos possuem diâmetro dentro desse intervalo, o que corresponde a $95 \%$ dos indivíduos analisados em Floresta Ombrófila Densa.

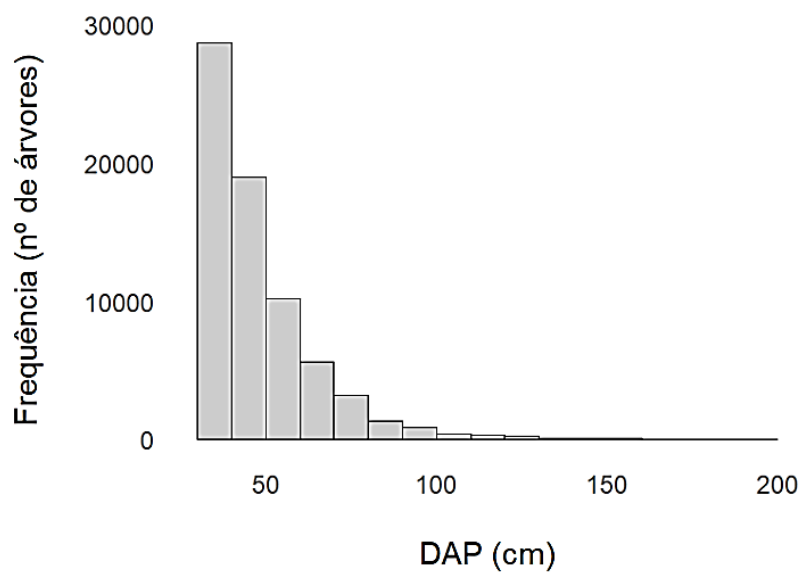

Figura 1. Distribuição dos indivíduos de Floresta Ombrófila Densa de acordo com os valores de DAP $(\mathrm{cm})$, para dados do Projeto RADAMBRASIL, no bioma Amazônia.

Nota-se que a distribuição dos indivíduos, de acordo com os valores dos diâmetros, é do tipo "j invertido" ou decrescente, característica de florestas naturais heterogêneas e multiâneas (MEYER, 1943). Esse comportamento de distribuição dos indivíduos também foi observado em áreas de Florestas Densas na Amazônia Central e no sul da Amazônia (OLIVEIRA et al., 2008; LIMA
\& LEÃO, 2013; SILVA et al., 2015). A densidade total foi de $60,77 \pm 16,98$ indivíduos. ha ${ }^{-1}$ e a área basal total $12,44 \pm$ $4,53 \mathrm{~m}^{2} \cdot$ ha $^{-1}$.

Cabe destacar que as árvores de maior porte (DAP $\geq 80$ $\mathrm{cm})$, apesar de menos frequentes, possuem importância tanto na diversidade ecológica da floresta quanto na atuação como sumidouros de carbono (SLIK et al., 2013; FAUSET et al., 2015).

Com relação à composição florística, em 1.158 hectares de Floresta Ombrófila Densa amostrados, constatou-se a ocorrência de 70.371 indivíduos, sendo 661 espécies distribuídas em 76 famílias. As famílias com maior número de indivíduos foram Sapotaceae (8.134 indivíduos), Lecythidaceae (6.373), Caesalpiniaceae (6.350) e Fabaceae (4.103). Ademais, as famílias Chrysobalanaceae, Burseraceae Moraceae e Lauraceae também foram representativas em relação ao número de indivíduos amostrados: 3.716; 3.150; 2.819; e 2.730, respectivamente. Essas oito famílias agruparam cerca de $44 \%$ do número total de indivíduos de analisados, em Floresta Densa.

Na Tabela 2, observa-se as 10 espécies mais importantes, com base nos valores de importância. Destaque para as espécies Eschweilera coriacea (DC.) S.A.Mori, Goupia glabra Aubl. e Pouteria caimito (Ruiz \& Pav.) Radlk., que apresentaram os maiores valores de importância para a área estudada.

Dentre essas 10 espécies, três pertencem à família Lecythidaceae e três à família Sapotaceae; uma, família Goupiaceae; uma, família Burseraceae; uma família Humiriaceae; e uma, família Lauraceae. Assim, pode-se observar que as famílias mais ocorrentes podem variar, conforme o tipo de análise conduzida, seja a partir do número total de indivíduos $(\mathrm{N})$ ou a partir de parâmetros fitossociológicos, como é o caso do valor de importância.

Também houve a constatação da ocorrência 89 espécies muito raras, cuja frequência é de um indivíduo por parcela, o que representa $13 \%$ das espécies de Floresta Densa estudadas. Essa porcentagem de espécies raras é considerada baixa, quando comparada a outros estudos, onde constatou-se a ocorrência de 40-60\% de espécies raras (OLIVEIRA \& AMARAL, 2004; SILVA et al., 2015). Como exemplo, pode-se citar as seguintes espécies consideradas raras: Fagara acreana K.Krause (Rutaceae), Chamaecrista orbiculata (Benth.) H.S.Irwin \& Barneby (Fabaceae), Ambelania acida Aubl. (Apocinaceae), Erythrina fusca Lour. (Fabaceae) e Hymenolobium petraeum Ducke (Fabaceae). Essas espécies diferem-se das encontradas nos estudos citados anteriormente, os quais foram conduzidos localmente, em poucas parcelas de inventário florestal: 20 
parcelas, de $500 \mathrm{~m}^{2}$ cada, no estudo de Oliveira \& Amaral (2004); 15 parcelas, de 1 ha cada, no estudo de Silva et al. (2015).

Essas diferenças na porcentagem de espécies raras podem ser explicadas pelo fato de que, quanto maior a abrangência dos levantamentos florestais - no presente caso, regional (todos os estados do bioma Amazônia) -, maior a chance de ocorrência de mais de um indivíduo por parcela nas áreas amostradas. Outro ponto que pode explicar o valor baixo de espécies raras refere-se ao período no qual os levantamentos foram realizados (décadas de 70 e 80), quando a Floresta Amazônica se encontrava menos perturbada e mais intacta do que nos dias atuais.

Tabela 2. Resultados dos parâmetros fitossociológicos da estrutura horizontal para as 10 espécies que apresentaram os maiores valores de importância (VI), a partir de dados de parcelas do Projeto RADAMBRASIL, em Floresta Ombrófila Densa.

\begin{tabular}{|c|c|c|c|c|c|c|c|c|c|c|}
\hline Espécie & Nome popular & $\mathbf{N}$ & $A B$ & DA & DR & DoA & DoR & FA & FR & VI \\
\hline Eschweilera coria & $\mathrm{N}$ & 2.574 & 39 & 2,22 & 3,67 & 0,34 & 2,77 & 63,3 & 1,94 & 2,8 \\
\hline Goupia glabra Aubl. & Cupiúba & .331 & 376,83 & 1,15 & 1,9 & 0,33 & 2,62 & 42,75 & 1,31 & 1,94 \\
\hline Pouteria caimito (Rui & Abil & 51 & 8 & 1,18 & 1,94 & 0,19 & 1,55 & 1,81 & 1,59 & 1,69 \\
\hline Bertholletia excelsa Bonpl. & Castanha-do-Brasil & 489 & 456,26 & 0,42 & 0,7 & 0,39 & 3,17 & 19 & 0,58 & 1,48 \\
\hline Protium altissimum (Aubl.) Ma & Breu-aroeira & 1.388 & 217,84 & 1,2 & 1,98 & 0,19 & 1,51 & 25,04 & 0,77 & 1,42 \\
\hline Cariniana micrantha Ducke & Castanha-de-r & 1.081 & 187,13 & 0,93 & 1,54 & 0,16 & 1,3 & 41,19 & 1,26 & 1,37 \\
\hline Pouteria bilocularis (H.K.A.WinkI.) Baehni & Abiu-casca-grossa & 1.048 & 187,51 & 0,91 & 1,49 & 0,16 & 1,3 & 39,98 & 1,23 & 1,34 \\
\hline Prieurella prieurii (A.DC.) Aubr. & Abiurana verr & 1.065 & 184,62 & 0,92 & 1,52 & 0,16 & 1,28 & 39,21 & 1,2 & 1,33 \\
\hline Sacoglottis guianensis Benth. & Uxirana & 1.004 & 180,49 & 0,87 & 1,43 & 0,16 & 1,25 & 42,92 & 1,32 & 1,33 \\
\hline Nectandra mollis (Kunth) Nees & Canela-ferrugem & 976 & 172,3 & 0,84 & 1,39 & 0,15 & 1,2 & 43,09 & 1,32 & 1,3 \\
\hline
\end{tabular}

Em que: $N=\mathrm{n}$ o de indivíduos; $A B$ = área basal $\left(\mathrm{m}^{2} . \mathrm{ha}^{-1}\right) ; \mathrm{DA}=$ densidade absoluta ( $\mathrm{n}$ - de indivíduos por hectare); $\mathrm{DR}=$ densidade relativa $(\%)$; DoA = dominância absoluta $\left(\mathrm{m}^{2}\right.$.ha-1); DoR = dominância relativa (\%); $F A=$ frequência absoluta $(\%) ; F R=$ frequência relativa $(\%) ;$ e $V I=$ valor de importância (\%).

O Índice de diversidade de Shannon-Wiener foi de 5,33 nats.ind ${ }^{-1}$, o qual corrobora com valores encontrados em florestas tropicais (KNIGHT, 1975) e indica uma alta riqueza de espécies nas parcelas de Floresta Densa analisadas. Além disso, esse valor corrobora com outros estudos realizados em regiões de Floresta Densa na Amazônia brasileira, como Oliveira \& Amaral (2004), que encontraram $\mathrm{H}^{\prime}$ de 5,01 nats.ind ${ }^{-1}$ em floresta na Amazônia Central. Já Alves \& Miranda (2008), obtiveram um valor de $\mathrm{H}^{\prime}$ de 4,25 nats.ind ${ }^{-1}$, na Amazônia Oriental.

Com relação aos valores de frequência absoluta (FA), as espécies Eschweilera coriacea, Goupia glabra, Pouteria caimito, Cariniana micrantha Ducke, Sacoglottis guianensis Benth. e Nectandra mollis (Kunth) Nees, apresentaram valores superiores a $40 \%$. Isso significa que, em 1 hectare de Floresta Densa, a chance de encontrar uma árvore dessas 6 espécies é de aproximadamente 63, 43, 52, 41, 43, e $43 \%$, respectivamente.

Ter Steege et al. (2013), em artigo publicado na revista Science, fizeram uma estimativa de que existem, aproximadamente, $16 \mathrm{mil}$ espécies de árvores na Amazônia. Porém, metade de todas as árvores pertence a apenas 227 espécies (cerca de apenas 1,4\%). Os autores denominaram essas 227 espécies como "hiperdominantes". Dentre essas espécies "hiperdominantes", estão Eschweilera coriacea, a qual apresentou o maior valor de importância nesse estudo), e Protium altissimum (Aubl.) Marchand, a quinta espécie com maior VI (Tabela 2).

Ademais, Ter Steege et al. (2013, Tabela S3) também estimaram que as famílias Lecythidaceae, Burseraceae e Moraceae são consideradas "hiperdominantes", corroborando com o os resultados do presente estudo, pois estas mesmas famílias apresentaram alta frequência de indivíduos e espécies.

Em estudo fitossociológico realizado em floresta densa, Silva et al. (2014) encontraram que a espécie Eschweilera coriacea apresentou maior valor de importância, e que as famílias Sapotaceae, Lecythidaceae e Burseraceae foram as mais ocorrentes, corroborando com os resultados do presente estudo.

A partir dos resultados aqui encontrados, observa-se que, ao comparar os resultados de levantamentos florísticos realizados nas décadas de 70 e 80 com levantamentos datados dos últimos 10 anos, semelhantes espécies e famílias continuam sendo consideradas como 
de ampla ocorrência na Floresta Amazônica.

\section{Floresta Ombrófila Aberta}

Na Floresta Ombrófila Aberta foram analisados 43.860 indivíduos (Tabela 1). Tal qual para Floresta Ombrófila Densa, a distribuição dos indivíduos, conforme os valores de DAP, também foi do tipo "j invertido", com maior proporção dos indivíduos nas classes de diâmetro $30-70 \mathrm{~cm}$ (cerca de 90\% dos indivíduos). A densidade total foi 51,36 $\pm 16,73$ indivíduos.ha- ${ }^{-1}$; a área basal total, 10,69 $\pm 4,35$ $\mathrm{m}^{2} \cdot \mathrm{ha}^{-1}$.

A ocorrência de indivíduos de grande porte (DAP $\geq 80$ $\mathrm{cm}$ ) pode indicar que, no momento em que os levantamentos florestais do Projeto RADAM foram realizados, não havia ocorrido grandes distúrbios recentes nas áreas analisadas. As espécies Ceiba pentandra (L.) Gaertn., Bertholletia excelsa Bonpl. e Dinizia excelsa Ducke (espécie com o maior DAP registrado em todas as parcelas, de $305 \mathrm{~cm}$ ) apresentaram indivíduos de grande porte.

A análise da riqueza em Floresta Aberta indicou a ocorrência de 593 espécies, distribuídas em 69 famílias. As famílias com maiores frequências de número de indivíduos foram: Caesalpiniaceae (5.090 indivíduos), Sapotaceae (3.502), Burseraceae (3.283), Fabaceae (3.060) e Moraceae (2.889). Ademais, destacaram-se as famílias Lecythidaceae,
Chrysobalanaceae, Euphorbiaceae e Lauraceae, com 2.141, 1.675, 1.609 e 1.519 indivíduos, respectivamente. Essas famílias agruparam $54 \%$ dos indivíduos de Floresta Aberta.

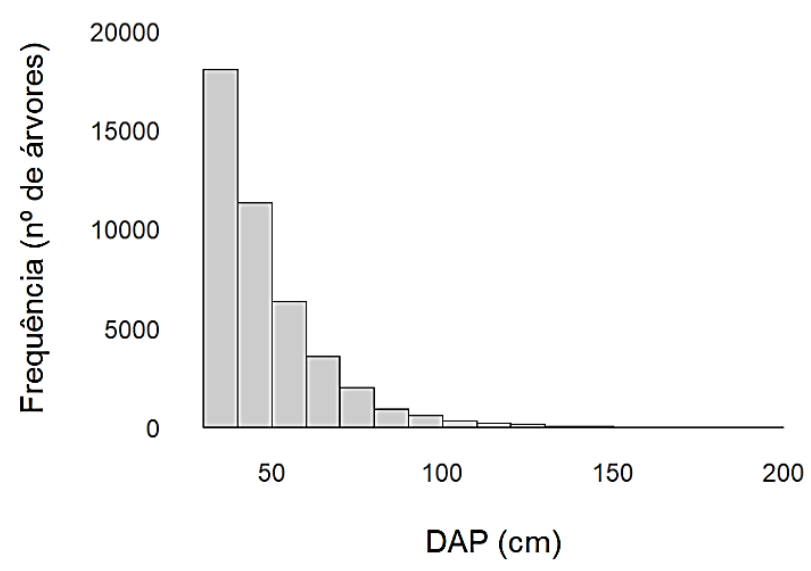

Figura 2. Distribuição dos indivíduos de Floresta Ombrófila Aberta de acordo com os valores de DAP $(\mathrm{cm})$, para dados do Projeto RADAMBRASIL no bioma Amazônia.

Na Tabela 3, observa-se as 10 espécies mais importantes nas parcelas estudadas, com base nos valores de importância (VI), com destaque para Protium altissimum, Eschweilera coriacea e Bertholletia excelsa, com maiores valores de importância para Floresta Aberta.

Tabela 3. Resultados dos parâmetros fitossociológicos da estrutura horizontal para as 10 espécies que apresentaram os maiores valores de importância (VI), a partir de dados de parcelas do Projeto RADAMBRASIL, em Floresta Ombrófila Aberta.

\begin{tabular}{|c|c|c|c|c|c|c|c|c|c|c|}
\hline Espécie & Nome popular & $\mathbf{N}$ & $A B$ & DA & DR & DoA & DoR & FA & FR & VI \\
\hline Protium altissimum (Aubl.) Marchand & Breu & 2.044 & 308,84 & 2,39 & 4,68 & 0,36 & 3,38 & 39,7 & 1,42 & 3,16 \\
\hline Eschweilera coriacea (DC.) S.A.Mori & Mat & 847 & 130,72 & 0,99 & 1,94 & 0,15 & 1,43 & 41,22 & 1,47 & 1,61 \\
\hline Bertholletia excelsa Bonpl. & $\begin{array}{c}\text { Castanha-do- } \\
\text { Brasil }\end{array}$ & 285 & 312,75 & 0,33 & 0,65 & 0,37 & 3,42 & 19,79 & 0,71 & 1,59 \\
\hline Dialium guianense (Aubl.) Sandwith & Tamarina & 648 & 111,05 & 0,76 & 1,48 & 0,13 & 1,22 & 35,95 & 1,28 & 1,33 \\
\hline Pseudolmedia multinervis Mildbr. & $\begin{array}{l}\text { Morácea- } \\
\text { chocolate }\end{array}$ & 653 & 90,05 & 0,76 & 1,49 & 0,11 & 0,99 & 38,52 & 1,38 & 1,29 \\
\hline Pouteria caimito (Ruiz \& Pav.) Radlk. & Abiu/abiurana & 561 & 91,89 & 0,66 & 1,28 & 0,11 & 1,01 & 36,07 & 1,29 & 1,19 \\
\hline Couratari pulchra Sandwith & Tauari & 359 & 158,33 & 0,42 & 0,82 & 0,19 & 1,73 & 27,75 & 0,99 & 1,18 \\
\hline Licania membranacea Sagot ex Laness. & Canela-branca & 651 & 84,6 & 0,76 & 1,49 & 0,1 & 0,93 & 28,34 & 1,01 & 1,14 \\
\hline Inga alba (Sw.) Willd. & Ingá-vermelha & 509 & 83,99 & 0,6 & 1,16 & 0,1 & 0,92 & 35,36 & 1,26 & 1,12 \\
\hline Hevea brasiliensis (Willd. ex A.Juss.) Müll.Arg. & Seringueira & 514 & 95,53 & 0,6 & 1,18 & 0,11 & 1,05 & 30,33 & 1,08 & 1,1 \\
\hline
\end{tabular}

Em que: $N=\mathrm{n}$ o de indivíduos; $A B$ = área basal $\left(\mathrm{m}^{2}\right.$.ha-1); $\mathrm{DA}=$ densidade absoluta ( $\mathrm{n}$ - de indivíduos por hectare); $\mathrm{DR}=$ densidade relativa $(\%) ;$ DoA = dominância absoluta $\left(\mathrm{m}^{2} \cdot\right.$ ha-1 $)$; DoR = dominância relativa (\%); $F A=$ frequência absoluta $(\%) ; F R=$ frequência relativa $(\%) ;$ e $V I=$ valor de importância (\%).

As famílias que concentraram as 10 espécies com maiores VI foram: Lecythidaceae (3 indivíduos), Fabaceae (2 indivíduos), Burseraceae (1), Moraceae (1), Sapotaceae
(1), Chrysobalanaceae (1) e Euphorbiaceae (1). Com maior frequência relativa, destacaram-se as espécies Eschweilera coriacea (41,22\%), Protium altissimum (39,7\%) e 
Pseudolmedia multinervis Mildbr. (38,52\%).

Tal qual para Floresta Densa, a análise de indivíduos em Floresta Aberta também constatou a ocorrência de espécies raras, tais como: Dilodendron bipinnatum Radlk. (Sapindaceae), Maclura tinctoria (L.) D.Don ex Steud. (Moraceae), Piptadenia moniliformis Benth. (Fabaceae), Apeiba tibourbou Aubl. (Malvaceae) e Sloanea latifolia (Rich.) K.Schum. (Elaeocarpaceae). Portanto, cerca de 14\% das espécies encontradas foram consideradas raras (81), em valor semelhante ao encontrado para Floresta Densa.

$O$ Índice de Shannon-Wiener encontrado foi de 5,35 nats.ind ${ }^{-1}$, valor semelhante ao encontrado para Floresta Densa. Portanto, apesar de ambientes de Floresta Aberta serem caracterizados por sub-bosque menos denso e menor ocupação de árvores por unidade de área, o valor de $\mathrm{H}^{\prime}$ revela a alta diversidade florística encontrada nas parcelas de Floresta Aberta aqui analisadas.

Cabe destacar que nos levantamentos florestais realizados no âmbito do Projeto RADAMBRASIL, espécies da família Arecaceae (palmeiras) não foram catalogadas. Essa família possui ampla ocorrência na bacia Amazônica. Além disso, segundo Ter Steege et al. (2013), a espécie Euterpe precatoria Mart. (açaí-do-amazonas) foi considerada a mais "hiperdominante" na Amazônia. Além disso, outra espécie da família Arecaceae, Euterpe oleraceae, foi a quinta mais "hiperdominante".

Isso pode ser explicado pelo fato de que os inventários florestais do Projeto RADAMBRASIL priorizaram espécies madeireiras e espécies de valor comercial na Amazônia. Dessa forma, o inventário florestal conduzido pelo RADAMBRASIL ainda se configuram como o maior já realizado na Amazônia brasileira. Além disso, os importantes resultados dos levantamentos florísticos oriundos desse Projeto - tal qual os aqui apresentados e discutidos - podem servir de subsídio para estudos correntes e futuros na Floresta Amazônica.

\section{CONCLUSÕES}

Uma alta diversidade de espécies foi observada para as tipologias Floresta Ombrófila Densa (76 famílias, 661 espécies) e Floresta Ombrófila Aberta (69 famílias, 593 espécies). Além disso, uma expressiva diversidade florística foi constatada a partir dos altos valores observados para o Índice de diversidade de Shannon-Wiener.

As espécies Eschweilera coriacea, Pouteria caimito e Protium altissimum, com altos valores de importância, foram comuns às tipologias florestais estudadas, destacando-se também pelos altos valores de densidade absoluta e frequência absoluta.
As famílias Lecythidaceae, Burseraceae e Moraceae foram representativas tanto em termos de número de indivíduos quanto número de espécies. Por serem consideradas famílias "hiperdominantes" na Amazônia, conclui-se que a diversidade florística dessa floresta, observada a partir de levantamento realizado nas décadas de 70 e 80, assemelha-se à diversidade encontrada em levantamentos datados dos últimos 10 anos.

\section{AGRADECIMENTOS}

Ao IBGE (Instituto Brasileiro de Geografia e Estatística), junto à plataforma BDiA (Banco de Dados de Informações Ambientais), pelo fornecimento dos dados. Ao CNPq (Conselho Nacional de Desenvolvimento Científico e Tecnológico) e à CAPES (Coordenação de aperfeiçoamento de pessoal de nível superior), pela concessão das bolsas de estudos que deram suporte à realização da presente pesquisa.

\section{REFERÊNCIAS}

ALVES, J.C.Z.O.; MIRANDA, I.D.S. Análise da estrutura de comunidades arbóreas de uma floresta amazônica de Terra Firme aplicada ao manejo florestal. Acta Amazonica, v.38, n.4, p.657666, 2008.

DALAGNOL, R. et al. Função para cálculo dos descritores fitossociológicos e similaridade entre sítios. 2016. Disponível em: https://github.com/ricds/fitoR

FAUSET, S. et al. Hyperdominance in Amazonian forest carbon cycling. Nature Communications, v.6, p.1-9, 2015.

HOPKINS, M.J.G. Modelling the known and unknown plant biodiversity of the Amazon Basin. Journal of Biogeography, v.34, n.8, p.1400-1411, 2007.

HOPKINS, M.J.G. Are we close to knowing the plant diversity of the Amazon? Anais da Academia Brasileira de Ciências, v.91, p.17, 2019.

INSTITUTO BRASILEIRO DE GEOGRAFIA E ESTATÍSTICA (IBGE). Banco de Dados de Informações Ambientais. 2019. Disponível em: https://bdiaweb.ibge.gov.br/\#/home.

KNIGHT, D.H.A. Phytosociological Analysis of Species-Rich Tropical Forest on Barro Colorado Island, Panama. Ecological Monographs, v.45, n.3, p.259-284, 1975.

LAMPRECHT, H. Ensayo sobre la estructura floristica de la parte Sur-Oriental del bosque universitario: El Caimital, Estado Barinas. Revista Forestal Venezolana, v.7, n.10/11, p.77-119, 1964.

LIMA, J.P.C.; LEÃO, J.R.A. Dinâmica de Crescimento e Distribuição Diamétrica de Fragmentos de Florestas Nativa e Plantada na Amazônia Sul Ocidental. Floresta e Ambiente, v.20, n.1, p.70-79, 2013. 
MACEDO, M.N. et al. Decoupling of deforestation and soy production in the southern Amazon during the late 2000s. Proceedings of the National Academy of Sciences of the United States of America, v.109, n.4, p.1341-1346, 2012.

MARTINS, F.R. Estrutura de uma floresta mesófila. [s.I.] Campinas: Editora da Universidade Estadual de Campinas, 1991.

MEYER, H.A. Management without rotation. Journal of Forestry, v.41, n.2, p.126-132, 1943.

MILLIKEN, W. et al. Amazon vegetation : how much don't we know and how much does it matter? Kew Bulletin, v.65, p.691709, 2011.

MUELLER, D.; ELLENBERG, H. Aims and Methods of Vegetation Ecology. New York: John Wiley and Sons, 1974.

NEPSTAD, D. et al. Slowing Amazon deforestation through public policy and interventions in beef and soy supply chains. Science, v.344, n.6188, p.1118-1123, 2014.

OLIVEIRA, A.N.; AMARAL, I.L. Florística e fitossociologia de uma floresta de vertente na Amazônia Central, Amazonas, Brasil. Acta Amazonica, v.34, n.1, p.21-34, 2004.

OLIVEIRA, A.N. et al. Composição e diversidade florísticoestrutural de um hectare de floresta densa de terra firme na Amazônia Central, Amazonas, Brasil. Acta Amazonica, v.38, n.4, p.627-641, 2008.

ORME, C.D.L. et al. Global hotspots of species richness are not congruent with endemism or threat. Nature, v. 436, n.18, p.10161019, 2005.

PINHEIRO, K.A.O. et al. Fitossociologia De Uma Área De Preservação Permanente No Leste Da Amazônia: Indicação De Espécies Para Recuperação De Áreas Alteradas. Floresta, v.37, n.2, p.175-187, 2007.

$R$ CORE TEAM. R: a language and environment for statistical computing, version 3.0.2. Vienna, Austria: R Foundation for Statistical Computing, 2019.

SANTANA, J.A.S. et al. Florística e fitossociologia em área de vegetação secundária na Amazônia oriental. Revista de Ciências Agrárias, n.41, p.105-120, 2004.

SHANNON, C.E.; WIENER, N. A mathematical theory of communication. Bell system technical journal, v.27, n.3, p.379423, 1948.

SILVA, K.E. et al. Composição florística e fitossociologia de espécies arbóreas do Parque Fenológico da Embrapa Amazônia Ocidental. Acta Amazonica, v.38, n.2, p.213-222, 2008.

SILVA, W.A.S. et al. Composição e Diversidade Florística em Um Trecho de Floresta de Terra Firme no Sudoeste do Estado do Amapá, Amazônia Oriental, Brasil. Biota Amazônia, v.4, n.3, p.3136, 2014.

SILVA, K.E. et al. Dinâmica florestal, estoque de carbono e fitossociologia de uma floresta densa de terra-firme na Amazônia Central. Scientia Forestalis, v.43, n.105, p.193-201, 2015.
SLIK, J.W.F. et al. Large trees drive forest aboveground biomass variation in moist lowland forests across the tropics. Global Ecology and Biogeography, v.22, n.12, p.1261-1271, 2013.

TER STEEGE, $H$. et al. Hyperdominance in the Amazonian tree flora. Science, v.342, n.6156, p.325-342, 2013.

TER STEEGE, H. et al. The discovery of the Amazonian tree flora with an updated checklist of all known tree taxa. Scientific Reports, v.6, p.1-15, 2016.

WICKHAM, H. ggplot2: elegant graphics for data analysis. 2.ed. Houston: Springer, 2016. 